ETH-TH/97-18

August 1997

\title{
Two-loop anomalous dimension in light-cone gauge with Mandelstam-Leibbrandt prescription
}

\author{
Gudrun Heinrich and Zoltan Kunszt巴 \\ Institute of Theoretical Physics, ETH, Zürich, Switzerland
}

\begin{abstract}
All the next-to-leading order results on Altarelli-Parisi splitting functions have been obtained in the literature either by using the operator product expansion method or by making use of the Curci Furmanski Petronzio (CFP) formalism in conjunction with light-like axial gauge, principal value (PV) prescription and dimensional regularization. In this paper we present the calculation of some non-singlet two-loop anomalous dimensions within the CFP formalism using light-cone axial gauge with Mandelstam-Leibbrandt (ML) prescription. We make a detailed comparison between the intermediate results given by the (PV) versus the (ML) method. We point out that the (ML) method is completely consistent and avoids the "phenomenological rules" used in the case of (PV) regularization.
\end{abstract}

\footnotetext{
${ }^{1}$ E-mails: gudrun@itp.phys.ethz.ch; kunszt@itp.phys.ethz.ch
} 


\section{Introduction}

At present and future high energy colliders a number of important hard scattering cross sections can be measured with high accuracy. The satisfactory description of these precision data requires the evaluation of next-to-leading order (NLO) and in few cases even next-to-next-to leading order (NNLO) corrections in perturbative QCD. A number of NLO corrections are by now available in the literature (see e.g. [1] and references therein). NNLO corrections, however, could be evaluated only in few cases. The special technique developed for the calculation of the NNNLO corrections to the total cross section of electron positron annihilation into hadrons [2] has recently been applied by Larin and Vermaseren [3] to get the NNLO and NNNLO anomalous dimensions for those operators which contribute to the Bjorken sum rule and the Gross-Llewellyn Smith sum rule.

In the case of hard processes with two initial hadrons, however, no complete NNLO result is available. Although the NNLO coefficient functions of the Drell-Yan process have been obtained in ref. [4], the phenomenological application of this result requires the calculation of the NNLO Altarelli-Parisi splitting functions (three-loop anomalous dimensions) as well. It seems that significant technical development has to be achieved before this calculation can be carried out.

Two rather different methods [5], [6, 7] have been used for the evaluation of the spin independent two-loop anomalous dimensions. Both methods recently also have been applied to the successful calculation of the NLO corrections of the spin dependent Altarelli-Parisi splitting functions [8, 9]. In the case of the first method [5] (OPE) one has to evaluate the overall ultraviolet divergences of twist-two local operator insertions. The results are obtained in moment space and the calculation can be carried out in Feynman gauge. Unfortunately, the number of the operator insertions increases very rapidly in higher orders and the treatment of operator mixing becomes increasingly cumbersome [11]. The second method [6, 7] (CFP) is based on the observation that in axial gauge the two-particle irreducible kernel of the ladder diagrams is finite. Therefore, using renormalization group properties, the anomalous dimensions are given by some projection of this kernel in agreement with the factorization theorem of mass singularities 13. The corrections to the Altarelli-Parisi splitting functions are obtained directly in configuration space by evaluating few Feynman diagrams. A detailed documentation of the NLO calculation using the

\footnotetext{
${ }^{2} \mathrm{~A}$ third method proposed in ref. [10] makes use of the the operator definition of the parton number densities and requires the evaluation of Feynam diagrams with attached eikonal factors. The method has not yet been tested in higher order calculations.
} 
CFP method appeared recently in ref. [12]. A theoretically questionable feature of the (CFP) method is the use of light-like axial gauge with principal value prescription. It is not clear whether the PV prescription remains valid in higher orders since standard quantization procedures in light-like axial gauge lead to the so-called Mandelstam-Leibbrandt (ML) prescription [14, 15]. Another technically unpleasant feature of the method is that the individual diagrams contributing to the finite 2PI kernel have soft and collinear singularities (which cancel in the sum). The actual algebraic complexity of the CFP method nevertheless is significantly smaller than the one of the OPE method, motivating the further study of its technical aspects. In particular, it is of interest to investigate the CFP method with ML prescription. A first attempt already appeared in the literature: The one-loop Altarelli-Parisi splitting functions have been calculated by Bassetto [19]. In the NLO calculation with ML prescription, the treatment of the ultraviolet part becomes more consistent. This is a clear principal advantage. However, the ML prescription is not without difficulties. Firstly, using two light-like vectors the integrals become more complicated. Secondly, the singular $1 / n q$ factor appearing in the expression for the axial gauge propagator is regularized with a $+i \epsilon$ prescription which results in new so-called axial ghost contributions.

In this paper we apply the CFP method with ML prescription to the calculation of non-singlet two-loop anomalous dimensions. In particular, we calculate all terms which are proportional to $C_{F}^{2}$. An interesting new feature of our calculation is the evaluation of the axial ghost contributions. In Section 2 we give a short review of the CFP formalism, in Section 3 the PV and ML prescriptions are discussed. Section 4 describes details of the calculation, treating separately the virtual and the real contributions. In Section 5 we make a crtitical comparison between the results obtained by using the PV and ML prescription and draw our conclusions from this discussion. An Appendix contains virtual integrals and phase space parametrizations.

\section{Framework of the calculation}

According to the factorization theorem, simple hard scattering cross sections can be written in perturbative QCD as convolutions of finite hard scattering cross sections and singular parton number densities

$$
\sigma\left(\frac{Q^{2}}{\mu^{2}}, \alpha_{S}\left(\mu^{2}\right), \epsilon\right)=\hat{\sigma}\left(\frac{Q^{2}}{\mu^{2}}, \alpha_{S}\left(\mu^{2}\right)\right) \otimes \Gamma\left(\alpha_{S}\left(\mu^{2}\right), \epsilon\right)
$$


where the symbol $\otimes$ indicates the convolution over longitudinal momentum fractions which in moment space is reduced to a simple product

$$
\sigma_{N}\left(\frac{Q^{2}}{\mu^{2}}, \alpha_{S}\left(\mu^{2}\right), \epsilon\right)=\hat{\sigma}_{N}\left(\frac{Q^{2}}{\mu^{2}}, \alpha_{S}\left(\mu^{2}\right)\right) \Gamma_{N}\left(\alpha_{S}\left(\mu^{2}\right), \epsilon\right)
$$

where

$$
f_{N}=\int_{0}^{1} d x x^{N-1} f(x)
$$

Our discussion will be valid for the non-singlet parton densities, but it can be easily generalized to the singlet case (matrix problem). The physical cross section $\sigma_{H}$ must be independent from $\mu^{2}$, therefore the deviation of the physical dimension of $\hat{\sigma}$ from its canonical value (anomalous dimension)

$$
\gamma_{N}\left(\alpha_{S}\left(\mu^{2}\right)\right)=-\frac{d}{d \ln \mu^{2}} \ln \hat{\sigma}_{N}\left(\frac{Q^{2}}{\mu^{2}}, \alpha_{S}\left(\mu^{2}\right)\right)
$$

is related to the moments of parton number densities

$$
\gamma_{N}\left(\alpha_{S}\left(\mu^{2}\right)=\frac{d}{d \ln \mu^{2}} \ln \Gamma_{N}\left(\alpha_{S}\left(\mu^{2}\right), \epsilon\right)=\beta\left(\alpha_{S}, \epsilon\right) \frac{d}{d \alpha_{S}} \ln \Gamma_{N}\left(\alpha_{S}\left(\mu^{2}\right), \epsilon\right)\right.
$$

where in the $\overline{\mathrm{MS}}$ scheme

$$
\beta\left(\alpha_{S}, \epsilon\right)=\frac{d \alpha_{S}}{d \ln \mu^{2}}=-\epsilon \alpha_{S}+\beta\left(\alpha_{S}\right)
$$

Expanding Eq. (5) in $\epsilon$ and keeping only the leading order term one gets

$$
\gamma_{N}\left(\alpha_{S}\left(\mu^{2}\right)=-\frac{d}{d \ln \alpha_{S}} \Gamma_{N}^{(1)}\left(\alpha_{S}\right)\right.
$$

where $\Gamma_{N}^{(1)}$ is the coefficient of $1 / \epsilon$ in the expansion of $\Gamma_{N}\left(\alpha_{S}\left(\mu^{2}\right), \epsilon\right)$

$$
\Gamma_{N}\left(\alpha_{S}\left(\mu^{2}\right), \epsilon\right)=1+\sum_{i} \frac{\Gamma_{N}^{(i)}\left(\alpha_{S}\right)}{\epsilon^{i}}
$$

In the parton picture, the physical hard scattering cross section $\sigma_{H}$ with incoming hadron $H$ is obtained by convoluting the partonic cross section $\hat{\sigma} \otimes \Gamma$ with "bare" parton densities $q^{B, H}$. The finite quantity $\left(\Gamma \otimes q^{B, H}\right)(x)=q_{f / H}\left(x, \mu^{2}\right)$ is then interpreted as the physical (renormalized) number density in the longitudinal momentum fraction $x$ of parton type $f$ in hadron $H$. This factorization scheme is shown diagrammatically in Figure 1. 


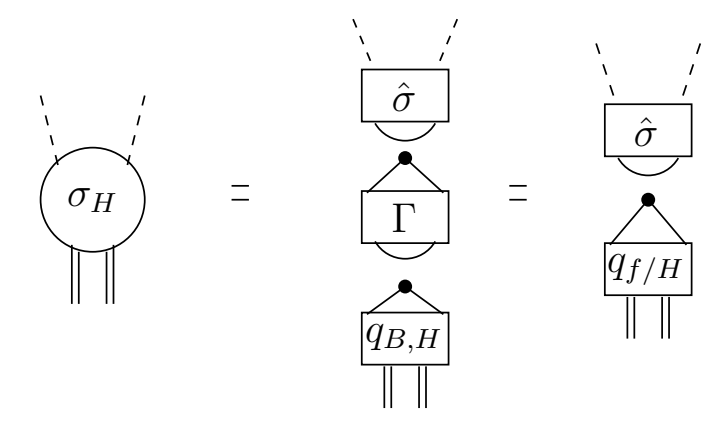

Figure 1: Factorization scheme

The parton evolution equation in moment space becomes simply

$$
\frac{d q_{N}\left(\mu^{2}\right)}{d \ln \mu^{2}}=\gamma_{N}\left(\alpha_{S}\right) q_{N}\left(\mu^{2}\right)
$$

and therefore the anomalous dimensions $\gamma_{N}\left(\alpha_{S}\right)$ defined through Eq. (4) can be interpreted as the moments of the Altarelli-Parisi splitting function

$$
\gamma_{N, q / q}\left(\alpha_{S}\right)=\int_{0}^{1} d x x^{N-1} P_{q / q}(x)
$$

where we consider the density of a quark within a quark. These relations allow to calculate $P_{q / q}\left(x, \alpha_{S}\right)$ in a power series in $\alpha_{S}$

$$
P_{q / q}\left(x, \alpha_{S}\right)=\left(\frac{\alpha_{S}}{2 \pi}\right) P_{q / q}^{(0)}(x)+\left(\frac{\alpha_{S}}{2 \pi}\right)^{2} P_{q / q}^{(1)}(x)+\mathcal{O}\left(\alpha_{S}{ }^{3}\right)
$$

which results in the following expansion of $\Gamma_{q / q}\left(x, \alpha_{S}, \epsilon\right)$

$$
\Gamma_{q / q}\left(x, \alpha_{S}, \epsilon\right)=\delta(1-x)-\frac{1}{\epsilon}\left\{\left(\frac{\alpha_{S}}{2 \pi}\right) P_{q / q}^{(0)}(x)+\frac{1}{2}\left(\frac{\alpha_{S}}{2 \pi}\right)^{2} P_{q / q}^{(1)}(x)+\ldots\right\}+\mathcal{O}\left(\frac{1}{\epsilon^{2}}\right) .
$$

Here $P_{q / q}^{(0)}(x)$ is the first order Altarelli-Parisi splitting function:

$$
P_{q / q}^{(0)}(x)=C_{F}\left(\frac{1+x^{2}}{1-x}\right)_{+}
$$

The CFP method for calculating $\Gamma_{q / q}\left(x, \alpha_{S}, \epsilon\right)$ in terms of a well-defined projection of the 2PI kernel $K_{0, a a^{\prime}}^{b b^{\prime}}\left(k, k^{\prime}\right)$ (upper lines are non-amputed, lower lines are amputed) of the ladder diagram

$$
\Gamma=\frac{1}{1-\mathcal{P} K}, \quad K=K_{0}\left(1-(1-\mathcal{P}) K_{0}\right)^{-1}
$$

consists in using a projector $\mathcal{P}=\mathcal{P}_{\epsilon} \otimes \mathcal{P}_{n}$ which can be considered as a pinching of the rungs of the ladder. This symbolic representation of the projector $\mathcal{P}$ indicates a projection acting on the $\epsilon$-dependence $\left(\mathcal{P}_{\epsilon}\right)$ and a projection acting on the spin, colour 
and momentum dependence $\mathcal{P}_{n}$ of the kernel. The rungs above the pinch are put on-shell and considered as external on-shell legs for the upper part of the diagram with averaging over spin and colour labels. The rungs below the pinch remain non-amputed. They have to be summed over the spin and colour indices in conjunction with the insertion of the operator $\frac{\not}{4 k n}$ for quarks and $-g_{\mu \nu}$ for gluons and have to be integrated over the off-shell loop momentum $k$. Finally, the projector extracts the single pole term of the whole expression, setting $\epsilon$ to zero in its residuum according to Eq. (8). The singular non-singlet quark density is therefore given by the expression

$$
\begin{aligned}
\Gamma_{q / q}\left(x, \alpha_{S}, \frac{1}{\epsilon}\right)= & Z_{F}\{\delta(1-x) \\
& \left.+P P\left\{x \int \frac{d^{m} k}{(2 \pi)^{m}} \delta\left(x-\frac{k n}{p n}\right) \operatorname{Trace}\left[\frac{\not n}{4 k n} \frac{K}{1-\mathcal{P} K} \not p\right]\right\}\right\}
\end{aligned}
$$

where $P P$ means to take the pole part. In fixed order perturbation theory we can expand $\Gamma-1=K /(1-\mathcal{P} K)$ in terms of the 2 PI kernel $K_{0}$ as

$$
\Gamma-1=\mathcal{P} K_{0}+\mathcal{P}\left(K_{0}\right)^{2}-\mathcal{P}\left(K_{0} \mathcal{P} K_{0}\right)+\ldots
$$

These equations are the basic ones to calculate the anomalous dimensions in higher orders. In two-loop order the contributions can be classified into virtual and real contributions. The virtual contributions are diagrams where only one internal parton line is cut and the diagrams have virtual subdiagrams. The real contributions are defined in terms of diagrams where two parton lines are cut and without any virtual subdiagram. In the CFP method it is convenient to treat separately the contribution from $Z_{F}$, the renormalization factor of the external leg, and hence to introduce the notation

$$
\begin{aligned}
\Gamma_{q / q}\left(x, \alpha_{S}, \frac{1}{\epsilon}\right)= & Z_{F} \hat{\Gamma}_{q / q}\left(x, \alpha_{S}, \frac{1}{\epsilon}\right) \\
\hat{\Gamma}_{q / q}\left(x, \alpha_{S}, \frac{1}{\epsilon}\right)= & \delta(1-x) \\
& -\frac{1}{\epsilon}\left\{\left(\frac{\alpha_{S}}{2 \pi}\right) \hat{P}_{q / q}^{(0)}(x)+\frac{1}{2}\left(\frac{\alpha_{S}}{2 \pi}\right)^{2} \hat{P}_{q / q}^{(1)}(x)+\mathcal{O}\left(\alpha_{S}^{3}\right)\right\}+\mathcal{O}\left(\frac{1}{\epsilon^{2}}\right) \\
Z_{F}= & 1-\frac{1}{\epsilon} \xi\left(\alpha_{S}\right)+\mathcal{O}\left(\frac{1}{\epsilon^{2}}\right) \\
P_{q / q}\left(x, \alpha_{S}\right)= & \hat{P}_{q / q}\left(x, \alpha_{S}\right)+\xi\left(\alpha_{S}\right) \delta(1-x) .
\end{aligned}
$$

An important simplification in the the CFP method is that one has to calculate only $\hat{P}_{a / b}^{(1)}(x)$ because the contribution of the wave function renormalization factor $Z_{F}$ at $x=1$ can easily be obtained from fermion number conservation and momentum conservation sum rules. 


\section{Principal value versus Mandelstam-Leibbrandt prescription}

As we already noted, a crucial ingredient of the CFP method is the use of the light-cone gauge (or light-like axial gauge), defined by

$$
n^{\mu} A_{\mu}^{a}(x)=0 \quad ; \quad n^{2}=0
$$

which formally leads to a gluon propagator of the form

$$
D_{\mu \nu}^{a b}(q)=\frac{-i \delta^{a b}}{q^{2}+i \epsilon}\left\{g_{\mu \nu}-\frac{n_{\mu} q_{\nu}+n_{\nu} q_{\mu}}{q n}\right\} .
$$

The 1/qn-factor in the gluon propagator gives rise to the so-called "spurious poles", singular terms in both the real and virtual contributions. Although these singular contributions must cancel in gauge invariant quantities, one has to use some regularization method for the evaluation of the individual contributions. As yet, in all calculations based on the CFP method, the principal value (PV) prescription has been used which is defined as

$$
\begin{aligned}
& \qquad \frac{1}{q n} \rightarrow \lim _{\delta \rightarrow 0} \frac{1}{2}\left(\frac{1}{q n+i \delta(p n)}+\frac{1}{q n-i \delta(p n)}\right)=\frac{q n}{(q n)^{2}+\delta^{2}(p n)^{2}} \\
& \text { where } \quad p^{2}=0 ; p n \neq 0 ; \vec{p}_{T}=\overrightarrow{0} .
\end{aligned}
$$

It is well-known [1 however that the PV prescription is incompatible with Wick rotation and hence power counting breaks down. Nevertheless, the spurious poles appearing as $\ln \delta, \ln ^{2} \delta$-terms in individual contributions cancel in the sum of all virtual and all real contributions. Another difficulty of the PV method is related to the ultraviolet renormalization constants which become dependent on longitudinal momentum fractions and on $\ln \delta$. CFP performed the ultraviolet renormalization by subtracting all ultraviolet poles, normal and spurious ones, calling this procedure "a phenomenological rule".

After the work of CFP it was pointed out that the principal value prescription is not consistent with canonical quantization in light-like axial gauge [20, 21. Correctly performed canonical quantization leads to the ML prescription defined as

$$
\frac{1}{q n} \rightarrow \lim _{\eta \rightarrow 0^{+}} \frac{q n^{*}}{q n q n^{*}+i \eta}
$$

\footnotetext{
${ }^{3}$ The subject of quantization and renormalization in noncovariant gauges and their use in perturbation theory is described in refs. 16, 17, 18.
} 
where the vector $n_{\mu}^{*}$ is "dual" to $n_{\mu}$ :

$$
n_{\mu}=\left(n_{0}, \vec{n}\right) ; n_{\mu}^{*}=\left(n_{0},-\vec{n}\right) ;\left(n^{*}\right)^{2}=0
$$

The ML prescription also appears in the literature as

$$
\frac{1}{q n} \rightarrow \lim _{\eta \rightarrow 0^{+}} \frac{1}{q n+i \eta \operatorname{sign}\left(q n^{*}\right)}
$$

In the present calculation, these alternative definitions lead to the same result. A crucial property of the ML prescription is that the spurious poles are placed in the complex $q_{0}$-plane in the same way as the "usual" covariant poles. Therefore, Wick rotation and power counting theorems for UV divergences remain valid. The propagator in the ML prescription can be decomposed into a term corresponding to the propagation of the physical polarizations and into a term which describes the propagation of scalar and longitudinal gluons in the $q n=0$ plane

$$
<0\left|T\left\{A_{\mu}^{a}(x) A_{\nu}^{b}(0)\right\}\right| 0>=<0\left|T\left\{T_{\mu}^{a}(x) T_{\nu}^{b}(0)\right\}\right| 0>+<0\left|T\left\{L_{\mu}^{a}(x) L_{\nu}^{b}(0)\right\}\right| 0>
$$

where

$$
\begin{aligned}
<0\left|T\left\{T_{\mu}^{a}(x) T_{\nu}^{b}(0)\right\}\right| 0>= & \frac{i \delta^{a b}}{(2 \pi)^{4}} \int \frac{d^{4} q e^{i q x}}{q^{2}+i \epsilon} \\
& \left(-g_{\mu \nu}+\frac{\left(n_{\mu} q_{\nu}+q_{\mu} n_{\nu}\right)}{q_{\perp}^{2}} \frac{2 q n^{*}}{n n^{*}}-\frac{n_{\mu} g_{0 \nu}+n_{\nu} g_{0 \mu}}{q_{\perp}^{2}} q^{2}\right)
\end{aligned}
$$

and

$$
\begin{aligned}
<0\left|T\left\{L_{\mu}^{a}(x) L_{\nu}^{b}(0)\right\}\right| 0>= & -\frac{i \delta^{a b}}{(2 \pi)^{4}} \int \frac{d^{4} q e^{i q x}}{q^{2}+q_{\perp}^{2}+i \epsilon} \\
& \left(\frac{\left(n_{\mu} q_{\nu}+q_{\mu} n_{\nu}\right)}{q_{\perp}^{2}} \frac{2 q n^{*}}{n n^{*}}-\frac{n_{\mu} g_{0 \nu}+n_{\nu} g_{0 \mu}}{q_{\perp}^{2}}\left(q^{2}+q_{\perp}^{2}\right)\right) .
\end{aligned}
$$

Adding up these contributions and using $q^{2}+q_{\perp}^{2}=2 q^{+} q^{-}=2\left(q n^{*}\right)(q n) / n n^{*}$ we get the axial-gauge propagator with ML regularization

$$
D_{\mu \nu}^{a b}(x)=\frac{i \delta^{a b}}{(2 \pi)^{4}} \int \frac{d^{4} q e^{i q x}}{q^{2}+i \epsilon}\left(-g_{\mu \nu}+\frac{\left(n_{\mu} q_{\nu}+q_{\mu} n_{\nu}\right) q n^{*}}{q n q n^{*}+i \epsilon}\right) .
$$

The discontinuity of this propagator can be decomposed into the physical axial-gauge contribution and an unphysical contribution

$$
\begin{aligned}
\operatorname{disc}\left[\frac{i d_{\mu \nu}(q)}{q^{2}}\right]= & 2 \pi \theta\left(q_{0}\right)\left\{-g_{\mu \nu}+\frac{2 q n^{*}}{n n^{*}} \frac{\left(n_{\mu} q_{\nu}+n_{\nu} q_{\mu}\right)}{q_{\perp}^{2}}\right\} \delta\left(q^{2}\right) \\
& -2 \pi \theta\left(q_{0}\right)\left\{\frac{2 q n^{*}}{n n^{*}} \frac{\left(n_{\mu} q_{\nu}+n_{\nu} q_{\mu}\right)}{q_{\perp}^{2}}\right\} \delta\left(q^{2}+q_{\perp}^{2}\right) .
\end{aligned}
$$


The term proportional to the delta function $\delta\left(q^{2}+q_{\perp}^{2}\right)$ is the so-called axial ghost contribution. It has been shown by Bassetto et al. [20] that all vectors of the physical Hilbert-space are annihilated by the creation operator of these degrees of freedom. Therefore, similarly to the Gupta-Bleuler ghosts of QED, they decouple from the S-matrix. The ghosts have negative mass squared, indefinite metric, they live in the $q n=0$ plane and their polarization sum is

$$
\sum_{\lambda=1,2} e_{\mu}^{\lambda}(q) e_{\nu}^{\lambda}(q)=-\frac{2 q n^{*}}{n n^{*}} \frac{\left(n_{\mu} q_{\nu}+q_{\mu} n_{\nu}\right)}{q_{\perp}^{2}} .
$$

In the ML prescription there is a cancellation between the standard axial gauge contribution and the axial ghost contribution: In the limit $q_{\perp}^{2} \rightarrow 0$ the spurious poles $1 / q_{\perp}^{2}$ appearing in the denominator of the polarization sums cancel each other as can be seen from Eq. (28). As a consequence, when contributions of diagrams with two cut gluon lines are calculated, the result must be independent from the regularization scheme of these spurious poles. One may use for example dimensional regularization or a PV regularization. The PV regularization can be chosen such that the standard axial gauge contribution from the first term in Eq. (28) becomes identical to the one of the PV calculation of CFP where no ghosts are included at all. Choosing the same PV regularization for the ghost part, one finds that the spurious poles cancel within the sum of all ghost diagrams, but a finite contribution is remaining from the ghost diagrams.

Using only dimensional regularization, mixed products of spurious, soft and collinear poles are obtained and one cannot see the details of the cancellation mechanism. In addition, one deals with poles of higher powers such that the integrals have to be calculated in higher order in the regularization parameter $\epsilon$. If we choose PV regularization, however, the various (spurious, soft, collinear) singular contributions can be clearly separated from each other. In particular, the result we get with ML prescription for the real contributions differs from the CFP result only because of the axial ghost contributions.

One can find a similar decomposition also for the virtual contributions with the help of a formula found in refs. 22, 23]. Let us consider the integral

$$
J_{n}^{A}\left(k_{1} \ldots k_{n-1}\right)=\int d^{m} q \frac{1}{q^{2}\left(q+k_{1}\right)^{2} \ldots\left(q+k_{n-1}\right)^{2} q n}
$$

defined in Appendix A. Using exponential parametrization for the propagator denominator factors and the ML prescription to regulate the $1 / q n$-denominator leads to

$$
\begin{aligned}
J_{n}^{A, \mathrm{ML}}\left(k_{1} \ldots k_{n-1}\right)= & \frac{1}{i^{n}} \int_{0}^{\infty} d a_{0} \ldots d a_{n-1} \exp \left\{i \sum a_{j} k_{j}^{2}-i R^{2} / z-z \epsilon\right\} \\
& \cdot \int d^{m} q \frac{\exp \left\{i z q^{2}\right\}}{q^{+}-R^{+} / z+i \eta \operatorname{sign}\left(q^{-}-R^{-} / z\right)}
\end{aligned}
$$




$$
\begin{aligned}
R^{\mu} & =a_{1} k_{1}^{\mu}+\ldots+a_{n-1} k_{n-1}^{\mu} \\
z & =a_{0}+\ldots+a_{n-1} .
\end{aligned}
$$

The $q$-integral

$$
J_{q}^{M L}=\int d q^{+} d q^{-} d^{(m-2)} \vec{q}_{\perp} \frac{\exp \left\{i z q^{2}\right\}}{q^{+}-R^{+} / z+i \eta \operatorname{sign}\left(q^{-}-R^{-} / z\right)}
$$

has been evaluated using the Cauchy theorem and integrating first over $d q^{+}$, then over $d q^{-}$. The integral over the transverse momenta is a standard Gaussian integral, and one obtains finally

$$
J_{q}^{M L}=\frac{-\pi^{\frac{m}{2}}(i z)^{1-\frac{m}{2}}}{R^{+}+i \eta z \operatorname{sign} R^{-}}\left\{1-\exp \left\{2 i R^{+} R^{-} / z-2 \eta\left|R^{-}\right|\right\}\right\}
$$

leading to

$$
\begin{aligned}
J_{n}^{A, \mathrm{ML}}\left(k_{1} \ldots k_{n-1}\right)= & \frac{-\pi^{\frac{m}{2}}}{i^{n+\frac{m}{2}-1}} \int_{0}^{\infty} d a_{0} \ldots d a_{n-1} \exp \left\{i \sum a_{j} k_{j}^{2}-z \epsilon\right\} \cdot z^{1-\frac{m}{2}} \\
& \cdot \frac{\exp \left\{-i R^{2} / z\right\}}{R^{+}+i \eta z \operatorname{sign} R^{-}}\left\{1-\exp \left\{2 i R^{+} R^{-} / z-2 \eta\left|R^{-}\right|\right\}\right\}
\end{aligned}
$$

whereas PV regularization leads to

$$
\begin{aligned}
J_{n}^{A, \mathrm{PV}}\left(k_{1} \ldots k_{n-1}\right)= & \frac{-\pi^{\frac{m}{2}}}{i^{n+\frac{m}{2}-1}} \int_{0}^{\infty} d a_{0} \ldots d a_{n-1} \exp \left\{i \sum a_{j} k_{j}^{2}-z \epsilon\right\} \cdot z^{1-\frac{m}{2}} \\
& \cdot \exp \left\{-i R^{2} / z\right\} \frac{R^{+}}{\left(R^{+}\right)^{2}+z^{2} \delta^{2}\left(p^{+}\right)^{2}}
\end{aligned}
$$

We can see from Eq. (33) that in the ML scheme there is a cancellation between the first and the second term as $R_{+} \rightarrow 0$. Therefore, the result will have no spurious poles and will be independent from the regularization method of the spurious singularity. Similar to the case of the real contributions, it is useful to use PV regularization for the individual spurious singularities even within the ML scheme. We emphasize that the application of a PV regularization here has nothing to do with the prescription required before carrying out the loop integral. Using PV regularization before doing the $d^{m} q$-integral leads to the result (34).

Comparing Eqs. (33) and (34) shows that the PV result can be identified as a subpart of the ML result by dropping the second term in Eq. (33) and taking the real part of the $1 /\left(R^{+}+i \eta z \operatorname{sign} R^{-}\right)$term. Hence the difference between the results for the virtual parts in the PV and ML prescription is given completely by the contributions from the 
second term in Eq. (33). It follows that the difference between the sum of all PV-and all ML-regulated virtual contributions must be equal to the sum of the real axial ghost contributions. That is indeed what we will find as a result of explicit calculation.

\section{Details of the calculation}

\subsection{Diagrams contributing to terms with colour factor $C_{F}^{2}$}

Since every colour structure defines a gauge invariant contribution we can simplify our exploratory study of the application of the ML prescription by considering only the terms that are proportional to $C_{F}^{2}$. Besides the obvious advantage of reducing the number of contributing Feynman diagrams, this colour structure has two additional simplifying features: i) the ultraviolet counter term is vanishing, ii) the sums of the real and virtual contributions are separately free from soft and collinear singularities. The axial-ghost contributions, however, remain important and therefore the $C_{F}^{2}$ structure gives a good opportunity to study these contributions in isolation from other complications. The contributing Feynman diagrams are listed in Fig. 2. The axial ghosts are denoted by dotted lines replacing cut gluon lines.

Topology (b)

$\sim C_{F}^{2}-\frac{1}{2} C_{F} N_{c}$

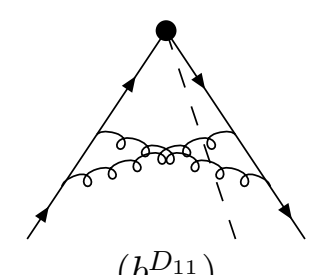

$\left(b^{D_{11}}\right)$
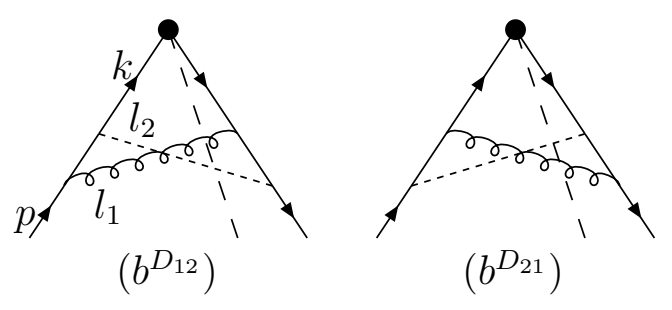

Topology (c) $\sim C_{F}^{2}-\frac{1}{2} C_{F} N_{c}$

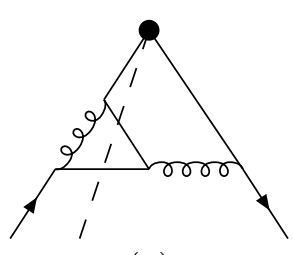

(c)
Topology (c) is equal in the ML and PV schemes since only quark lines are cut 

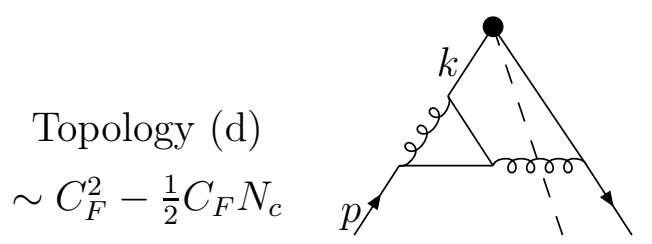

(d)

Topology (h)

$$
\sim C_{F}^{2}
$$
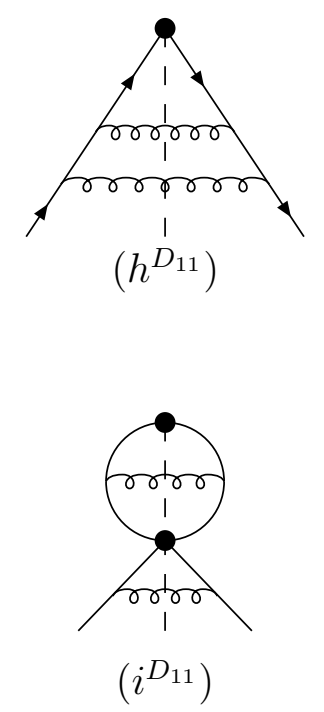

Topology (i)

$$
\sim C_{F}^{2}
$$
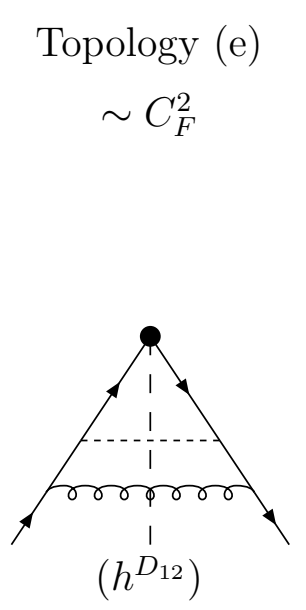
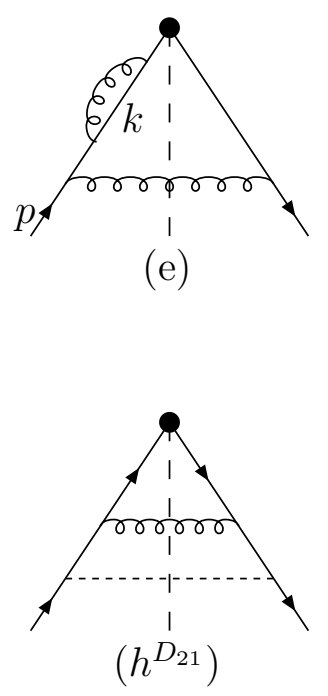

(e)

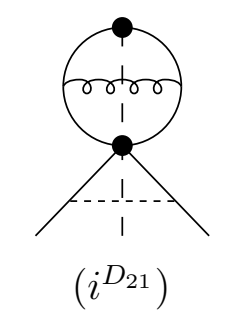

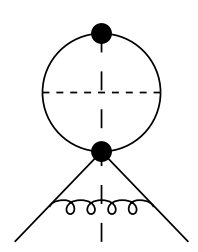

$\left(i^{D_{12}}\right)$

Figure 2: Diagrams contributing to $\hat{\Gamma}_{q / q}^{(1)}(x)$ with colour factor $C_{F}^{2}$

\subsection{Virtual corrections}

\subsubsection{Quark selfenergy}

The quark selfenergy (see topology (e) in Fig. 2) can be split into two terms

$$
\begin{aligned}
\Sigma & =\Sigma^{F}+\Sigma^{A} \\
& =C_{F} g^{2} \int \frac{d^{m} q}{(2 \pi)^{m}} \frac{\gamma_{\mu}(\not k+\not q) \gamma_{\nu}}{(k+q)^{2} q^{2}}\left\{-g_{\mu \nu}+\frac{q_{\mu} n_{\nu}+q_{\nu} n_{\mu}}{q n}\right\}
\end{aligned}
$$

where $\Sigma^{F}$ denotes that part of the expression which stems from the Feynman part (the part proportional to $-g_{\mu \nu}$ ) of the gluon propagator, $\Sigma^{A}$ denotes the remaining axial part. 
$\Sigma^{F}$ can be written in terms of simple loop integrals as

$$
\Sigma^{F}=g^{2} C_{F} \frac{(m-2)}{(2 \pi)^{m}}\left(\not k J_{2}^{F}(k)+\gamma^{\nu} J_{2 \nu}^{F}(k)\right)
$$

where

$$
\begin{aligned}
J_{2}^{F}(k) & =\int d^{m} q \frac{1}{q^{2}(q+k)^{2}}=Q_{\epsilon}^{k} T_{0} \quad ; \quad T_{0}=\frac{1}{\epsilon_{U V}}+2 \\
J_{2 \mu}^{F}(k) & =\int d^{m} q \frac{q_{\mu}}{q^{2}(q+k)^{2}}=-Q_{\epsilon}^{k} T_{1} k^{\mu} ; T_{1}=\frac{1}{2} T_{0} \\
Q_{\epsilon}^{k} & =i \pi^{\frac{m}{2}} \Gamma(1+\epsilon)\left(-k^{2}\right)^{-\epsilon} .
\end{aligned}
$$

We use the notation $\epsilon_{U V}$ for $\epsilon$ when the singular contribution comes from the ultraviolet region. The axial part $\Sigma^{A}$ is given by

$$
\Sigma^{A}=-g^{2} C_{F} \frac{1}{(2 \pi)^{m}}\left(2 k^{2} \not h J_{2}^{A}(k)+\not h J_{2 \mu}^{A}(k) \gamma^{\mu} \not k+\not k J_{2 \mu}^{A}(k) \gamma^{\mu} \not h\right)
$$

where

$$
\begin{aligned}
& J_{2}^{A}(k)=\int d^{m} q \frac{1}{q^{2}(q+k)^{2} q n}=-\frac{Q_{\epsilon}^{k}}{k n} P_{0}(k) \\
& J_{2 \mu}^{A}(k)=\int d^{m} q \frac{q_{\mu}}{q^{2}(q+k)^{2} q n}=\frac{Q_{\epsilon}^{k}}{k n}\left(P_{1}(k) k^{\mu}+P_{2}(k) p^{\mu}+P_{3}(k) n^{\mu}\right) .
\end{aligned}
$$

The actual values of the form factors $P_{0}, P_{1}, P_{2}, P_{3}$ are different for the PV and the ML prescription and can be found in Appendix A. In terms of form factors we obtain

$$
\begin{aligned}
\Sigma^{F} & =C_{F} \frac{g^{2}}{(2 \pi)^{m}} Q_{\epsilon}^{k} \frac{(m-2)}{2} \not k T_{0} \\
\Sigma^{A} & =-C_{F} \frac{g^{2}}{(2 \pi)^{m}} \frac{Q_{\epsilon}^{k}}{k n}\left(2 \not h k^{2}\left(P_{1}(k)-P_{0}(k)\right)+P_{2}(k)(\not h \not p \not k+\not k \not p \not h)\right) \\
& =-C_{F} \frac{g^{2}}{(2 \pi)^{m}} \frac{Q_{\epsilon}^{k}}{k n}\left(2 \not h k^{2}\left(P_{1}(k)-P_{0}(k)\right)+2 P_{2}(k)\left(\not k p n-\not p k n+\frac{k^{2}}{2} \not h\right)\right)
\end{aligned}
$$

Then the contribution of topology (e) to the parton density can be written asf (see Eq. (14))

$$
\hat{\Gamma}_{q / q}^{(1, e)}(x, \epsilon)=P P\left\{\frac{1}{16 \pi^{2}} \frac{(4 \pi)^{\epsilon}}{\Gamma(1-\epsilon)} \int_{0}^{Q^{2}} d\left(-k^{2}\right)\left(-k^{2}\right)^{-1-\epsilon} x(1-x)^{-\epsilon} \cdot 2 T^{(e)}\left(x, k^{2}, \epsilon\right)\right\}
$$

\footnotetext{
${ }^{4}$ Note that a factor of two has to be included for diagrams which are not symmetric.
} 
where

$$
\begin{aligned}
T^{(e)}\left(x, k^{2}, \epsilon\right)= & C_{F} \frac{i g^{2}}{\left(k^{2}\right)^{2}} \operatorname{Tr}\left[\frac{\not h}{4 k n} \not k \Sigma \not k \gamma^{\mu} \not p \gamma^{\nu} \not k\right] d_{\mu \nu}(p-k) \\
= & -C_{F}^{2} \alpha_{S}^{2}(4 \pi)^{\epsilon} \Gamma(1+\epsilon)\left(-k^{2}\right)^{-\epsilon} \\
& \cdot \frac{2}{x}\left\{\hat{P}_{q / q}(x, \epsilon)\left[T_{0}(\epsilon-1)+4\left(P_{1}(k)-P_{0}(k)\right)+\frac{2}{x} P_{2}(k)\right]\right. \\
& \left.+2 P_{2}(k) \frac{(1+x)}{1-x}\right\} .
\end{aligned}
$$

Details about the phase space integral are given in Appendix B.

$\hat{P}_{q / q}(x, \epsilon)$ is the leading order splitting function in $m=4-2 \epsilon$ dimensions

$$
\hat{P}_{q / q}(x, \epsilon)=\frac{1+x^{2}}{1-x}-\epsilon(1-x) .
$$

\subsubsection{Vertex correction}

The contribution from diagram (d) can also be split into a Feynman part and an axial part

$$
\Gamma_{\mu, b}=\Gamma_{\mu, b}^{F}+\Gamma_{\mu, b}^{A}
$$

which can written in terms of form factors of simple scalar, vector and tensor loop integrals given in Appendix A. Inserting this expression into Eq. (14) and using a definition for $T^{(d)}$ similar to the one we used for diagram (e) we obtain for the Feynman part

$$
\begin{aligned}
T^{\left(d_{F}\right)}\left(x, k^{2}, \epsilon\right)= & -C_{F}\left(C_{F}-\frac{N_{c}}{2}\right) \alpha_{S}^{2}(4 \pi)^{\epsilon} \Gamma(1+\epsilon)\left(-k^{2}\right)^{-\epsilon} \\
& \cdot \frac{4}{x}\left\{\left\{R_{1}-R_{0}+(2-\epsilon) R_{2}-(1-\epsilon)\left(R_{4}+R_{5}\right)\right\}[x+\epsilon(1-x)]\right. \\
& \left.+2 R_{6}(1-\epsilon)^{2} \hat{P}_{q / q}(x, \epsilon)\right\}
\end{aligned}
$$

and for the axial part

$$
\begin{aligned}
T^{\left(d_{A}\right)}\left(x, k^{2}, \epsilon\right)= & -C_{F}\left(C_{F}-\frac{N_{c}}{2}\right) \alpha_{S}^{2}(4 \pi)^{\epsilon} \Gamma(1+\epsilon)\left(-k^{2}\right)^{-\epsilon} \\
& \cdot\left\{\frac{4}{x}\left(\left(P_{0}(k)-P_{1}(k)\right) \hat{P}_{q / q}(x, \epsilon)-P_{2}(k) \frac{1+x}{1-x}\right)\right. \\
& +\left(p^{2} / k^{2}\right)^{-\epsilon} \frac{4}{x}\left(P_{0}(p)-P_{1}(p)-P_{2}(p)\right) \hat{P}_{q / q}(x, \epsilon) \\
& +\frac{2}{x}\left[\left(S_{1}+S_{4}+(2-x) S_{2}-2 S_{0}+R_{0}\right) \hat{P}_{q q}(x, \epsilon)\right. \\
& \left.\left.-2\left(R_{1}+R_{2}-R_{0}\right) \frac{1+x}{1-x}\right]\right\} .
\end{aligned}
$$

The form factors $R_{i}, P_{j}, S_{l} ; i=0, \ldots, 6 ; j=0, \ldots, 2 ; l=0, \ldots 4$ are defined and given in Appendix A both for PV and ML prescription. 


\subsubsection{Ultraviolet renormalization}

We use dimensional regularization to regulate both the ultraviolet and infrared singularities. Since we do first the loop integrals we must assume that in $m-4 \epsilon, \epsilon$ is positive. After adding the counter terms for the quark selfenergy and vertex one-loop subdiagrams we obtain an ultraviolet finite answer. Then we can analytically continue the result to negative values of $\epsilon$ and we can go on with the evaluation of the Feynman parameter integrals. First we should keep $p$ off-shell and set it on-shell only after ultraviolet renormalization. It is known that the sum of the two ultraviolet counter terms for the vertex and quark selfenergy diagrams in regular gauges vanishes as a consequence of abelian gauge invariance. Keeping only the ultraviolet pole part of the form factors $T_{0}, R_{6}, P_{i}$ (see Appendix A) and using Eqs. (42,45,46) one can see that the sum of the ultraviolet contributions is zero in the ML case, but is non-vanishing in the PV case. In particular one finds

$$
\begin{aligned}
T_{U V}^{(e), M L} & =C_{F}^{2} \alpha_{S}{ }^{2} \frac{1}{\epsilon_{U V}} \frac{2}{x}\left[-3 p_{q q}(x)+2\right] \\
T_{U V}^{(d), M L} & =C_{F}\left(C_{F}-\frac{N_{c}}{2}\right) \alpha_{S}{ }^{2} \frac{1}{\epsilon_{U V}} \frac{2}{x}\left[3 p_{q q}(x)-2\right] \\
T_{U V}^{(e), P V} & =C_{F}^{2} \alpha_{S}{ }^{2} \frac{1}{\epsilon_{U V}} \frac{2}{x} p_{q q}(x)\left[-3+4 I_{0}+4 \ln x\right] \\
T_{U V}^{(d), P V} & =C_{F}\left(C_{F}-\frac{N_{c}}{2}\right) \alpha_{S}{ }^{2} \frac{1}{\epsilon_{U V}} \frac{2}{x} p_{q q}(x)\left[3-4 I_{0}-2 \ln x\right] \\
p_{q q}(x) & =\frac{1+x^{2}}{1-x} \\
\text { and so } & \\
T_{\mathrm{UV}}^{(e+d), \mathrm{ML}} & =0 \\
T_{\mathrm{UV}, \mathrm{C}, \mathrm{PV}}^{(e+d), \mathrm{PV}} & =C_{F}^{2} \alpha_{S}{ }^{2} \frac{1}{\epsilon_{U V}} \frac{4}{x} p_{q q}(x) \ln x .
\end{aligned}
$$

This is an essential difference between the PV and ML schemes. The leftover UV singularity (47) in the PV scheme is a remainder from the contributions of the spurious poles to the UV renormalization constants. In the ML prescription, there are also additional terms

which are even not proportional to the Born term $p_{q q}(x)$, stemming from the $n^{*}$-part of the virtual integrals, but those terms completely cancel. Therefore it does not matter in the ML case whether they are subtracted or not. In the PV case however, the leftover term in (47) leads to an additional contribution to the anomalous dimension in the following way: Subtracting all UV poles, normal and spurious ones, means that in the external loop 
integralf over $k^{2}, T^{(e+d), P V}$ is replaced by its subtracted value

$$
T_{\mathrm{R}}^{(e+d), \mathrm{PV}}=T^{(e+d), \mathrm{PV}}-T_{\mathrm{UV}}^{(e+d), \mathrm{PV}} .
$$

Note that $T^{(e+d), \mathrm{PV}}$ contains a factor of $\left(-k^{2}\right)^{-\epsilon}$ whereas $T_{\mathrm{UV}}^{(e+d), \mathrm{PV}}$ does not. Inserting the UV subtracted expression (48) into the $k^{2}$-integral (41) and disregarding the finite parts of $T_{\mathrm{R}}^{(e+d), \mathrm{PV}}$ is leading to

$$
\begin{aligned}
& P P\left\{\frac{1}{16 \pi^{2}} \frac{(4 \pi)^{\epsilon}}{\Gamma(1-\epsilon)} \int_{0}^{Q^{2}} d\left(-k^{2}\right)\left(-k^{2}\right)^{-1-\epsilon} x(1-x)^{-\epsilon} \cdot 2 T_{\mathrm{R}}^{(e+d), \mathrm{PV}, \text { sing }}\right\} \\
= & C_{F}^{2}\left(\frac{\alpha_{S}}{2 \pi}\right)^{2}(1-x)^{-\epsilon} \int_{0}^{Q^{2}} d\left(-k^{2}\right)\left(-k^{2}\right)^{-1-\epsilon}\left[\left(-k^{2}\right)^{-\epsilon} \hat{P}_{q / q}(x, \epsilon) 2 \frac{\ln x}{\epsilon}-p_{q q}(x) 2 \frac{\ln x}{\epsilon}\right] \\
= & \frac{1}{2} C_{F}^{2}\left(\frac{\alpha_{S}}{2 \pi}\right)^{2}\left(\frac{2 \ln x}{\epsilon^{2}} p_{q q}(x)+\frac{2 \ln x}{\epsilon}\left[1-x+p_{q q}(x) \ln (1-x)\right]+\mathcal{O}\left(\epsilon^{0}\right)\right) .
\end{aligned}
$$

The double pole term has to be dropped since $\hat{\Gamma}_{q / q}\left(x, \alpha_{S}, \epsilon\right)$ is defined through simple poles in $\epsilon$ (see Eq. (16)). But the single pole term gives a contribution to $\hat{\Gamma}_{q / q}\left(x, \alpha_{S}, \epsilon\right)$ which is exactly the difference of the virtual contributions (PV-ML) virt obtained by using PV

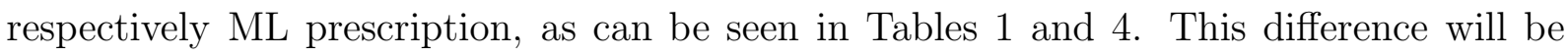
compensated by the ghost diagrams present only in the ML case, as will be explained in Section 4.3 .

The complete contributions of the virtual diagrams to $\hat{\Gamma}_{q / q}^{(1)}(x, \epsilon)$ are listed in Table 1 . Virtual diagrams where the cut line is a ghost line would give a contribution at $x=1$ only, so they do not contribute to $\hat{\Gamma}_{q / q}^{(1)}(x, \epsilon)$ (see Eqs. (16), (17)).

\subsection{Real contributions}

In this section we give some details concerning the calculation of the contributions from the diagrams of topologies (b),(c),(h) and (i). Topology (i) represents the subtraction terms $\mathcal{P}\left(K_{0} \mathcal{P} K_{0}\right)$ in Eq. (15) and consists of two Born diagrams linked by an additional projection.

\footnotetext{
${ }^{5}$ We can consider the last integral over $k^{2}$ as either infrared or ultraviolet divergent, depending on where we put a cutoff. CFP treat its divergence as a collinear infrared singularity, using an ultraviolet cutoff $Q^{2}$. Collins and Soper [10] instead introduce an infrared cutoff and consider the last integral as an ulraviolet divergent integral.
} 


\begin{tabular}{|c|c|c|c|c|c|c|}
\hline \multicolumn{7}{|c|}{ Finite part } \\
\hline & $e_{M L}$ & $d_{M L}$ & $e_{P V}$ & $d_{P V}$ & $(e+d)_{M L}$ & $(e+d)_{P V}$ \\
\hline$p_{q q}(x)$ & 6 & -6 & 7 & -7 & 0 & 0 \\
\hline$p_{q q}(x) \ln ^{2} x$ & 0 & 0 & -2 & 2 & 0 & 0 \\
\hline$p_{q q}(x) \ln x \ln (1-x)$ & 0 & 0 & -4 & 2 & 0 & -2 \\
\hline$p_{q q}(x) \ln x$ & -1 & 1 & 0 & 0 & 0 & 0 \\
\hline$p_{q q}(x) \ln (1-x)$ & 3 & -3 & 3 & -3 & 0 & 0 \\
\hline$p_{q q}(x) L i_{2}(1-x)$ & 4 & -2 & 0 & 2 & 2 & 2 \\
\hline$p_{q q}(x) \pi^{2} / 3$ & -2 & 2 & -2 & 2 & 0 & 0 \\
\hline$x /(1-x)$ & 2 & -2 & 0 & 0 & 0 & 0 \\
\hline$x$ & 0 & 1 & -3 & 4 & 1 & 1 \\
\hline 1 & -4 & 4 & 3 & -3 & 0 & 0 \\
\hline$x \ln x$ & -1 & 1 & 4 & -2 & 0 & 2 \\
\hline $\ln x$ & 1 & -1 & -4 & 2 & 0 & -2 \\
\hline $\ln (1-x)$ & -2 & 2 & 0 & 0 & 0 & 0 \\
\hline \multicolumn{7}{|c|}{ Spurious poles } \\
\hline$p_{q q}(x) I_{0} \ln (1-x)$ & 0 & 0 & -4 & 4 & 0 & 0 \\
\hline$p_{q q}(x) I_{0} \ln x$ & 0 & 0 & -4 & 4 & 0 & 0 \\
\hline$p_{q q}(x) I_{0}$ & 0 & 0 & 0 & 0 & 0 & 0 \\
\hline$p_{q q}(x) I_{1}$ & 0 & 0 & 4 & -4 & 0 & 0 \\
\hline$x I_{0}$ & 0 & 0 & 4 & -4 & 0 & 0 \\
\hline$I_{0}$ & 0 & 0 & -4 & 4 & 0 & 0 \\
\hline \multicolumn{7}{|c|}{ Singular part } \\
\hline$p_{q q}(x) I_{0} / \epsilon_{U V}$ & 0 & 0 & 4 & -4 & 0 & 0 \\
\hline$p_{q q}(x) \ln x / \epsilon_{U V}$ & 0 & 0 & 4 & -2 & 0 & 2 \\
\hline$p_{q q}(x) / \epsilon_{U V}$ & -3 & 3 & -3 & 3 & 0 & 0 \\
\hline $1 / \epsilon_{U V}$ & 2 & -2 & 0 & 0 & 0 & 0 \\
\hline
\end{tabular}

Table 1: The results for the virtual diagrams after UV subtraction. The virtual contribution to $\hat{\Gamma}_{q / q}^{(1)}(x, \epsilon)$ is obtained as a sum of the finite entries of this table multiplied by $-\frac{1}{2 \epsilon} C_{F}^{2}\left(\frac{\alpha_{S}}{2 \pi}\right)^{2}$. The singular part shows the ultraviolet counter terms. 


\subsubsection{Standard contribution}

The typical integral we have to evaluate for topologies (b),(c) and (h) can be written as

$$
I^{(a)}=\frac{1}{2} \frac{g^{4}}{(2 \pi)^{m}} \int \frac{d k^{2}}{k^{4}} d^{(m-2)} \vec{k} \int d \Phi\left(l_{1}, l_{2}\right) \cdot M^{(a)}\left(x, l_{1}, l_{2}, k, \epsilon\right)
$$

where $d \Phi\left(l_{1}, l_{2}\right)$ is the two-body-phase space for the two cut lines and $M^{(a)}$ the matrix element for topology (a). In terms of light-cone variables $q_{\mu}=\left(q^{+}, q^{-}, \vec{q}\right)$

$$
\begin{aligned}
d \Phi\left(l_{1}, l_{2}\right)= & \frac{4 \pi^{2}}{(2 \pi)^{m}} \int d l_{1}^{+} d l_{2}^{+} d l_{1}^{-} d l_{2}^{-} d^{(m-2)} \vec{l}_{1} d^{(m-2)} \vec{l}_{2} \delta\left(p^{+}-k^{+}-l_{1}^{+}-l_{2}^{+}\right) \\
& \cdot \delta^{(m-2)}\left(\vec{k}+\vec{l}_{1}+\vec{l}_{2}\right) \delta\left(k^{-}+l_{1}^{-}+l_{2}^{-}\right) \delta\left(l_{1}^{2}\right) \delta\left(l_{2}^{2}\right) .
\end{aligned}
$$

Introducing dimensionless parameters $z_{j}=l_{j}^{+} / p^{+}, j=1,2$ and using the relation $q^{-}=\left(q^{2}+\vec{q}^{2}\right) /\left(2 q^{+}\right)$on gets

$$
\begin{aligned}
d \Phi\left(l_{1}, l_{2}\right)= & \frac{2 \pi^{2}}{(2 \pi)^{m}} \int \frac{d z_{1}}{z_{1}} \frac{d z_{2}}{z_{2}} d \vec{l}_{1} d \vec{l}_{2} \delta\left(1-x-z_{1}-z_{2}\right) \\
& \cdot \delta\left(\vec{k}+\overrightarrow{l_{1}}+\overrightarrow{l_{2}}\right) \delta\left(\frac{k^{2}}{x}+\frac{\vec{k}^{2}}{x}+\frac{\vec{l}_{1}^{2}}{z_{1}}+\frac{\vec{l}_{2}^{2}}{z_{2}}\right) .
\end{aligned}
$$

The external loop also requires the integration over $d \vec{k}$. The transverse momentum integrals can be carried out easily if we introduce new momenta such that i) the $\delta$-function will be diagonal, ii) the propagator denominators appearing in the matrix element become as simple as possible. The appropriate choice is different for different topologies. For example, for toplogy (b) and (h) the good variables are

$$
\vec{l}_{1}=\vec{h}_{1} \quad \vec{l}_{2}=-\frac{z_{2}}{1-z_{1}}\left(\vec{h}_{1}+\vec{h}_{2}\right) \quad \vec{h}_{1} \vec{h}_{2}=h_{1} h_{2} \cos \theta
$$

For the phase space integral we obtain the form

$$
\begin{aligned}
P S= & \frac{2 \pi^{4-2 \epsilon}}{(2 \pi)^{m}} \frac{1}{\Gamma^{2}(1-\epsilon)}\left(-k^{2}\right)^{1-2 \epsilon} \int_{0}^{1-x} d z_{1}\left(\frac{z_{1}\left(1-x-z_{1}\right)}{x}\right)^{-\epsilon} \\
& \cdot \int_{0}^{1} d u u^{-\epsilon}(1-u)^{-\epsilon} \cdot \frac{\Gamma(1-\epsilon)}{\sqrt{\pi} \Gamma\left(\frac{1}{2}-\epsilon\right)} \int_{0}^{\pi} d \theta(\sin \theta)^{-2 \epsilon}
\end{aligned}
$$

where $P S$ is defined as $P S=\int d \vec{k} d \Phi\left(l_{1}, l_{2}\right)$. The azimuthal dependence of the denominator is either trivial or it can be cast into the form $1 /\left(1+\lambda^{2}+2 \lambda \cos \theta\right)$, such that we need the integrals

$$
I_{A}=\frac{\Gamma(1-\epsilon)}{\sqrt{\pi} \Gamma\left(\frac{1}{2}-\epsilon\right)} \int_{0}^{\pi} d \theta(\sin \theta)^{-2 \epsilon}=1
$$




$$
\begin{aligned}
& I_{C}\left(\lambda^{2}\right)=\frac{\Gamma(1-\epsilon)}{\sqrt{\pi} \Gamma\left(\frac{1}{2}-\epsilon\right)} \int_{0}^{\pi} d \theta \frac{(\sin \theta)^{-2 \epsilon}}{1+\lambda^{2}+2 \lambda \cos \theta} \\
& I_{C}\left(\lambda^{2}\right)= \begin{cases}F\left(1,1+\epsilon ; 1-\epsilon, \lambda^{2}\right) & \text { for } \lambda^{2}<1 \\
\frac{1}{\lambda^{2}} F\left(1,1+\epsilon ; 1-\epsilon, \frac{1}{\lambda^{2}}\right) & \text { for } \lambda^{2}>1 .\end{cases}
\end{aligned}
$$

In the case of topology (b) for example one has

$$
\begin{aligned}
\lambda^{2} & =\frac{b u}{1-u} \\
b & =\frac{z_{1}\left(1-x-z_{1}\right)}{x}
\end{aligned}
$$

such that the integration range for the $u$-integration will be split at $u=\frac{1}{1+b}$ according to Eq. (53).

Carrying out these integrals one should keep the regularization parameter of the PV scheme appearing in Eq. (20) to regularize the spurious poles. As we explained above, the contibutions from diagrams $(c),\left(b^{D_{11}}\right),\left(h^{D_{11}}\right)$ and $\left(i^{D_{11}}\right)$ are the same for the PV and the ML scheme. There are no ghosts related to diagram (c) since it does not contain cut gluon lines. The individual contributions are shown in Tables 2 and 3 . The new feature of the ML scheme is that we should also add the contributions from the axial ghost terms (see Eq. (28) and Fig. 2).

\subsubsection{Ghost contributions}

The diagrams considered in this section are given by topologies $b^{D_{12}}, h^{D_{12}}, h^{D_{21}}, i^{D_{12}}$ and $i^{D_{21}}$. Topology $b^{D_{21}}$ leads to the same result as $b^{D_{12}}$ because the diagrams are symmetric under exchange of $l_{1} \leftrightarrow l_{2}$. Diagrams with two cut ghost lines only give a contribution at $x=1$, which has been omitted since it can be obtained more easily from fermion number conservation (see Section 2).

The phase space for diagrams with one ghost line and one gluon line cut is given by (for details see Appendix B.2)

$$
\begin{aligned}
P S^{g h o s t}= & F_{\epsilon}\left|k^{2}\right|^{2-2 \epsilon} x^{-1+\epsilon}(1-x)^{-\epsilon} \int_{0}^{1} d u u^{-\epsilon}(1-u)^{1-\epsilon} \int_{0}^{1} d y y^{-\epsilon} \\
& \frac{1}{B\left(\frac{1}{2}-\epsilon, \frac{1}{2}-\epsilon\right)} \int_{0}^{1} d w[w(1-w)]^{-\frac{1}{2}-\epsilon} .
\end{aligned}
$$

The results for the ghost diagrams are given in Tables 2 and 3 . Table 14 shows the results for both, the real and the virtual part in the PV and ML schemes, where real means standard plus ghost contributions in the ML case. 


\begin{tabular}{|c|c|c|c|c|c|c|c|c|c|c|c|c|}
\hline & \multicolumn{4}{|c|}{ no ghosts } & \multicolumn{6}{|c|}{ ghosts } & \multirow{2}{*}{$\begin{array}{c}\text { no ghosts } \\
(b+h-i)_{11}\end{array}$} & \multirow{2}{*}{$\frac{\text { ghosts }}{(b+h-i)_{g h}}$} \\
\hline & $b^{D_{11}}$ & $(c)$ & $h^{D_{11}}$ & $i^{D_{11}}$ & $b^{D_{12}}$ & $b^{D_{21}}$ & $h^{D_{12}}$ & $h^{D_{21}}$ & $i^{D_{12}}$ & $i^{D_{21}}$ & & \\
\hline$p_{q q}(x) \ln ^{2}(1-x)$ & 0 & 0 & 6 & 6 & 0 & 0 & 0 & 0 & 0 & 0 & 0 & 0 \\
\hline$p_{q q}(x) \ln ^{2} x$ & -1 & -1 & 0 & -2 & $1 / 2$ & $1 / 2$ & 1 & 0 & 2 & 0 & 1 & 0 \\
\hline$p_{q q}(x) \ln x \ln (1-x)$ & 0 & 0 & -2 & -2 & 0 & 0 & -2 & 0 & 0 & 0 & 0 & -2 \\
\hline$p_{q q}(x) \ln (1-x)$ & 4 & 0 & -4 & 0 & & & & & & & 0 & 0 \\
\hline$p_{q q}(x) \ln x$ & 0 & $-3 / 2$ & 0 & 0 & & & & & & & 0 & 0 \\
\hline$p_{q q}(x) L i_{2}(1-x)$ & 0 & -2 & 0 & 0 & 1 & 1 & -2 & 0 & 0 & 0 & 0 & 0 \\
\hline$p_{q q}(x) \pi^{2} / 3$ & 0 & 0 & -2 & -2 & & & & & & & 0 & 0 \\
\hline$x \ln ^{2} x$ & 0 & 0 & $-1 / 2$ & 0 & & & & & & & $-1 / 2$ & 0 \\
\hline $\ln ^{2} x$ & 0 & 0 & $-1 / 2$ & 0 & & & & & & & $-1 / 2$ & 0 \\
\hline$x \ln x$ & 2 & $-7 / 2$ & -4 & 0 & 0 & 0 & 2 & 0 & 0 & 0 & -2 & 2 \\
\hline $\ln x$ & 2 & $-7 / 2$ & 0 & -2 & 0 & 0 & -2 & 0 & 0 & 0 & 4 & -2 \\
\hline$(1+x) L i_{2}(1-x)$ & 0 & 0 & 2 & 2 & & & & & & & 0 & 0 \\
\hline $1-x$ & 0 & -7 & 1 & -2 & & & & & & & 3 & 0 \\
\hline 1 & 0 & -1 & 0 & 0 & & & & & & & 0 & 0 \\
\hline$x^{2} \ln x \ln (1-x) /(1-x)$ & 0 & 0 & -4 & -4 & & & & & & & 0 & 0 \\
\hline \multicolumn{13}{|c|}{ Spurious poles } \\
\hline$p_{q q}(x) I_{0}$ & 4 & 0 & 0 & 4 & -2 & -2 & 0 & $\overline{0}$ & 0 & -4 & 0 & 0 \\
\hline$p_{q q}(x) I_{0} \ln x$ & 0 & 0 & -4 & -4 & 0 & 0 & 2 & 2 & 4 & 0 & 0 & 0 \\
\hline$p_{q q}(x) I_{0} \ln (1-x)$ & 0 & 0 & 4 & 4 & 0 & 0 & -2 & -2 & 0 & -4 & 0 & 0 \\
\hline$p_{q q}(x) I_{1}$ & 0 & 0 & 4 & 4 & 0 & 0 & -2 & -2 & -4 & 0 & 0 & 0 \\
\hline$I_{0} \cdot x /(1-x)$ & 0 & 0 & -8 & -8 & 0 & 0 & 4 & 4 & 0 & 8 & 0 & 0 \\
\hline
\end{tabular}

Table 2: Results for real diagrams. The contribution to $\hat{\Gamma}_{q / q}^{(1)}(x, \epsilon)$ is obtained as a sum of the entries multiplied by $-\frac{1}{2 \epsilon} C_{F}^{2}\left(\frac{\alpha_{S}}{2 \pi}\right)^{2}$ 


\begin{tabular}{|c|c|c|c|c|c|c|c|c|c|c|c|c|}
\hline & \multicolumn{4}{|c|}{ no ghosts } & \multicolumn{6}{|c|}{ ghosts } & \multirow{2}{*}{$\frac{\text { no ghosts }}{(b+h-i)_{11}}$} & \multirow{2}{*}{$\frac{\text { ghosts }}{(b+h-i)_{g h}}$} \\
\hline & $b^{D_{11}}$ & $(c)$ & $h^{D_{11}}$ & $i^{D_{11}}$ & $b^{D_{12}}$ & $b^{D_{21}}$ & $h^{D_{12}}$ & $h^{D_{21}}$ & $i^{D_{12}}$ & $i^{D_{21}}$ & & \\
\hline$p_{q q}(x) I_{0} / \epsilon$ & 0 & 0 & -4 & -4 & 0 & 0 & 2 & 2 & 2 & 2 & 0 & 0 \\
\hline$p_{q q}(x) \ln x / \epsilon$ & 0 & 0 & 0 & 0 & 0 & 0 & 2 & 0 & 2 & 0 & 0 & 0 \\
\hline$p_{q q}(x) \ln (1-x) / \epsilon$ & 0 & 0 & -4 & -4 & 0 & 0 & 0 & 0 & 0 & 0 & 0 & 0 \\
\hline$(1+x) \ln x / \epsilon$ & 0 & 0 & -1 & -1 & 0 & 0 & 0 & 0 & 0 & 0 & 0 & 0 \\
\hline$(1-x) / \epsilon$ & 0 & 0 & 2 & 2 & 0 & 0 & 0 & 0 & 0 & 0 & 0 & 0 \\
\hline
\end{tabular}

Table 3: Real diagrams, singular parts 


\begin{tabular}{|c|c|c|c|c|c|c|c|}
\hline & \multicolumn{2}{|c|}{ real sum } & \multirow{2}{*}{$\begin{array}{c}\operatorname{diff}_{\text {real }} \\
(\mathrm{ML}-\mathrm{PV})_{\text {real }}\end{array}$} & \multicolumn{2}{|c|}{ virtual sum } & \multirow{2}{*}{$\frac{\operatorname{diff}_{\text {virt }}}{(\mathrm{ML}-\mathrm{PV})_{\text {virt }}}$} & \multirow[b]{2}{*}{$\begin{array}{c}\text { sum of ghosts } \\
\text { only }\end{array}$} \\
\hline & $(b+h-i)_{M L}$ & $(b+h-i)_{P V}$ & & $(e+d)_{M L}$ & $(e+d)_{P V}$ & & \\
\hline$p_{q q}(x) \ln ^{2} x$ & 1 & 1 & 0 & 0 & 0 & 0 & 0 \\
\hline$p_{q q}(x) \ln x \ln (1-x)$ & -2 & 0 & -2 & 0 & -2 & 2 & -2 \\
\hline$p_{q q}(x) L i_{2}(1-x)$ & 0 & 0 & 0 & 2 & 2 & 0 & 0 \\
\hline$x \ln ^{2} x$ & $-1 / 2$ & $-1 / 2$ & 0 & 0 & 0 & 0 & 0 \\
\hline $\ln ^{2} x$ & $-1 / 2$ & $-1 / 2$ & 0 & 0 & 0 & 0 & 0 \\
\hline$x \ln x$ & 0 & -2 & 2 & 0 & 2 & -2 & 2 \\
\hline $\ln x$ & 2 & 4 & -2 & 0 & -2 & 2 & -2 \\
\hline 1 & 3 & 3 & 0 & 0 & 0 & 0 & 0 \\
\hline$x$ & -3 & -3 & 0 & 1 & 1 & 0 & 0 \\
\hline
\end{tabular}

Table 4: Comparison of real and virtual parts 


\section{Discussion and Conclusions}

The reevaluation of the two-loop anomalous dimension in light-like axial gauge is a good testing ground to understand the usefulness and reliability of this gauge. The success of the calculation of Curci, Furmanski and Petronzio using the principal value prescription for the $1 / n q$ factor is based on some heuristic treatment of the ultraviolet renormalization. Since the anomalous dimensions are related to the ultraviolet renormalization of bilocal operators, it is important to provide a better field theoretical support for the "phenomenologica rules" found by CFP. Within the PV scheme one gets spurious UV singular terms which are subtracted. The Mandelstam-Leibbrandt prescription eliminates this difficulty and is in addition consistent with canonical quantization. Therefore it has to be expected that the straightforward use of the Feynman rules as given by the ML scheme naturally provides a field theoretically correct treatment, leading to a deeper understanding of the heuristic CFP rules. This expectation has been justified by our explicit calculation of the two-loop anomalous dimensions proportional to the colour factor $C_{F}^{2}$.

It is instructive to make a detailed comparison of the evaluations in the PV and ML schemes. The necessary informations are summarized in Tables 1, 2, 3 and 4 . In evaluating one-loop insertions in the ML-scheme, the spurious poles cancel within the loop integrals, such that the ML result is free from spurious singularities, whereas the PV integrals can be recovered as a subpart of the ML integrals, this subpart being plagued by spurious singularities (see Eq. (34) and Table 1). After having carried out the momentum integration with ML prescription, one can regularize the spurious poles of Feynman-parameter integrals with a PV prescription. If all singularities are regularized with dimensional regularization the answer for the sum remains the same. The sums of all virtual contributions are different in the two schemes and the difference is due to the difference in the ultraviolet subtraction.

The differences in the real contributions can be organized according to Eq. (28). The first term in this equation is the standard axial gauge contribution. The second term defines the axial ghost contribution. The results are summarized in Tables 2 , 3 and 4 . The sum of all real contributions is different in the two schemes, the difference being given by the axial ghost contribution which compensates the difference found in the virtual corrections. We see again that the individual terms in the ML scheme are more regular. If we combine the contributions of the diagrams $\left(b^{D_{11}}, b^{D_{12}}, b^{D_{21}}\right)$ or $\left(h^{D_{11}}, h^{D_{12}}, h^{D_{21}}\right)$ or $\left(i^{D_{11}}, i^{D_{12}}, i^{D_{21}}\right)$ their sum is separately finite. In addition, the sums of the ghost and non-ghost diagrams are also separately finite. The fact that the spurious singularities 
cancel separately in the sum of the virtual and real contributions is a consequence of gauge invariance.

In summary, the evaluation of the two-loop anomalous dimension in the ML scheme is a consistent method. The axial ghost contributions are important to get the correct answer. It is remarkable that the phenomenological rule of CFP for subtracting all ultraviolet contributions (spurious and non-spurious ones) leads to the same additional terms as provided by the axial ghost contributions in the ML scheme.

It would be interesting to see the differences between the two schemes and the higher consistency of the ML scheme also for the remaining colour structures. 


\section{A Virtual integrals}

We define general n-point integrals, containing no axial denominator $1 / q n$, by

$$
J_{n}^{F \mu_{1} \ldots \mu_{s}}\left(k_{1} \ldots k_{n-1}\right)=\int d^{m} q \frac{q^{\mu_{1}} \ldots q^{\mu_{s}}}{q^{2}\left(q+k_{1}\right)^{2} \ldots\left(q+k_{n-1}\right)^{2}}
$$

and n-point integrals containing one axial denominator $1 / q n$ by

$$
\begin{aligned}
J_{n}^{A \mu_{1} \ldots \mu_{s}}\left(k_{1} \ldots k_{n-1}\right) & =\int d^{m} q \frac{q^{\mu_{1}} \ldots q^{\mu_{s}}}{q^{2}\left(q+k_{1}\right)^{2} \ldots\left(q+k_{n-1}\right)^{2} q n} \\
I_{n}^{A \mu_{1} \ldots \mu_{s}}\left(k_{1} \ldots k_{n}\right) & =\int d^{m} q \frac{q^{\mu_{1}} \ldots q^{\mu_{s}}}{\left(q+k_{1}\right)^{2} \ldots\left(q+k_{n}\right)^{2} q n}
\end{aligned}
$$

In the calculation of the contribution of the diagrams of type (e) and (d) we need two-point integrals and three-point integrals.

\section{A.1 Two-point integrals}

First we introduce a general parametrization of the integrals as follows

$$
\begin{aligned}
J_{2}^{F}(r) & =Q_{\epsilon}^{r} T_{0} \quad ; \quad T_{0}=\frac{1}{\epsilon_{U V}}+2 \\
J_{2}^{F \mu}(r) & =-Q_{\epsilon}^{r} T_{1} r^{\mu} ; \quad T_{1}=\frac{1}{2} T_{0} \\
J_{2}^{A}(r) & =-\frac{Q_{\epsilon}^{r}}{r n} P_{0}(r) \\
J_{2 \mu}^{A}(r) & =\frac{Q_{\epsilon}^{r}}{r n}\left\{P_{1}(r) r_{\mu}+P_{2}(r) n_{\mu}^{*}+P_{3}(r) n_{\mu}\right\} \\
Q_{\epsilon}^{r} & =i \pi^{\frac{m}{2}} \Gamma(1+\epsilon)\left(-r^{2}\right)^{-\epsilon} .
\end{aligned}
$$

The results for the form factors $P_{0}, P_{1}, P_{2}$ and $P_{3}$ depend on the regularization of the axial denominator $1 / q n$.

\section{A.1.1 Form factors for the ML scheme}

If we use ML prescription we get

$$
\begin{aligned}
& P_{0}^{M L}(r)=L i_{2}(1)-L i_{2}\left(1-\chi_{r}\right) \\
& P_{1}^{M L}(r)=-\frac{\chi_{r} \ln \chi_{r}}{1-\chi_{r}}
\end{aligned}
$$




$$
\begin{aligned}
& P_{2}^{M L}(r)=\frac{r n}{n n^{*}}\left(\frac{1}{\epsilon_{U V}}+2+\frac{\chi_{r} \ln \chi_{r}}{1-\chi_{r}}\right) \\
& P_{3}^{M L}(r)=\frac{r n^{*}}{n n^{*}}\left(-1+\frac{\ln \chi_{r}}{1-\chi_{r}}+\frac{1}{\chi_{r}}\left[L i_{2}(1)-L i_{2}\left(1-\chi_{r}\right)\right]\right)
\end{aligned}
$$

where $\chi_{r}$ is defined as

$$
\chi_{r}=: \frac{2 r n r n^{*}}{n n^{*} r^{2}}
$$

\section{A.1.2 Form factors for the PV scheme}

In the case of PV prescription on gets

$$
\begin{aligned}
& P_{0}^{P V}(r)=\frac{I_{0}}{\epsilon_{U V}}+\frac{\ln \left(r^{+}\right)}{\epsilon_{U V}}-I_{1}+I_{0} \ln \left(r^{+}\right)+\frac{1}{2} \ln ^{2}\left(r^{+}\right)+L i_{2}(1) \\
& P_{1}^{P V}(r)=\frac{1}{\epsilon_{U V}}+2 \\
& P_{2}^{P V}(r)=0 \\
& P_{3}^{P V}(r)=\frac{r^{2}}{2 r n}\left\{\frac{I_{0}}{\epsilon_{U V}}+\frac{\ln \left(r^{+}\right)}{\epsilon_{U V}}-\frac{2}{\epsilon_{U V}}-I_{1}+I_{0} \ln \left(r^{+}\right)+\frac{1}{2} \ln ^{2}\left(r^{+}\right)-4+L i_{2}(1)\right\}
\end{aligned}
$$

There is no $n^{*}$-dependence in this case and we used the definitions

$$
\begin{aligned}
r^{+} & =\frac{r n}{p n} \\
I_{0} & =\int_{0}^{1} d u \frac{u}{u^{2}+\delta^{2}}=-\ln \delta+\mathcal{O}(\delta) \\
I_{1} & =\int_{0}^{1} d u \frac{u \ln u}{u^{2}+\delta^{2}}=-\frac{1}{2} \ln ^{2} \delta-\frac{1}{4} L i_{2}(1)+\mathcal{O}(\delta) .
\end{aligned}
$$

\section{A.2 Two-point integrals for special momenta $k$ and $p$}

The parameters for the two-point integrals with the special kinematics used in the calculation can be read off from relations (55) to (62). In this case

$$
\begin{aligned}
p & =n^{*} \\
(p-k)^{2} & =0 \Rightarrow p k=\frac{k^{2}}{2} \quad ; \quad k_{T}^{2}=-k^{2}(1-x) \quad ; \quad k^{2}<0 \\
k^{+} & =x \quad ; \quad p^{+}=1 \\
\chi_{k} & =\frac{2 k n k n^{*}}{n n^{*} k^{2}}=x \\
\chi_{p} & =\frac{2 p^{+} p^{-}}{p^{2}}=1 \quad \text { for } p^{2} \neq 0, p_{T}^{2}=0 .
\end{aligned}
$$


Note also that

$$
\lim _{\chi_{p} \rightarrow 1} \frac{\chi_{p} \ln \chi_{p}}{1-\chi_{p}}=-1
$$

Since the integrals $J_{2}^{A}(p)$ and $J_{2 \mu}^{A}(p)$ only contribute to the UV counterterm, $p^{2}$ has to be off-shell in this case. In the infrared region, where $\epsilon<0$ and $p^{2}=0$, the integrals $J_{2}^{A}(p)$ and $J_{2 \mu}^{A}(p)$ vanish due to the overall factor $\left(-p^{2}\right)^{-\epsilon}$.

\section{A.2.1 Form factors in the ML scheme}

$$
\begin{aligned}
& P_{0}^{M L}(k)=L i_{2}(1)-L i_{2}(1-x) \\
& P_{1}^{M L}(k)=-\frac{x \ln x}{1-x} \\
& P_{2}^{M L}(k)=x\left\{\frac{1}{\epsilon_{U V}}+2+\frac{x \ln x}{1-x}\right\} \\
& P_{3}^{M L}(k)=\frac{k^{2}}{2 p n}\left\{-1+\frac{\ln x}{1-x}+\frac{1}{x}\left[L i_{2}(1)-L i_{2}(1-x)\right]\right\} \\
& P_{0}^{M L}(p)=L i_{2}(1) \\
& P_{1}^{M L}(p)=1 \\
& P_{2}^{M L}(p)=\frac{1}{\epsilon_{U V}}+1 \\
& P_{3}^{M L}(p)=\frac{p^{2}}{2 p n}\left\{-2+L i_{2}(1)\right\}
\end{aligned}
$$

\section{A.2.2 Form factors in the PV scheme}

$$
\begin{aligned}
& P_{0}^{P V}(k)=\frac{I_{0}}{\epsilon_{U V}}+\frac{\ln x}{\epsilon_{U V}}-I_{1}+I_{0} \ln x+\frac{1}{2} \ln ^{2} x+L i_{2}(1) \\
& P_{1}^{P V}(k)=\frac{1}{\epsilon_{U V}}+2 \\
& P_{2}^{P V}(k)=0 \\
& P_{3}^{P V}(k)=\frac{k^{2}}{2 k n}\left\{\frac{I_{0}}{\epsilon_{U V}}+\frac{\ln x}{\epsilon_{U V}}-\frac{2}{\epsilon_{U V}}-I_{1}+I_{0} \ln x+\frac{1}{2} \ln ^{2} x-4+L i_{2}(1)\right\} \\
& P_{0}^{P V}(p)=\frac{I_{0}}{\epsilon_{U V}}-I_{1}+L i_{2}(1) \\
& P_{1}^{P V}(p)=\frac{1}{\epsilon_{U V}}+2 \\
& P_{2}^{P V}(p)=0
\end{aligned}
$$




$$
P_{3}^{P V}(p)=\frac{p^{2}}{2 p n}\left\{\frac{I_{0}}{\epsilon_{U V}}-\frac{2}{\epsilon_{U V}}-I_{1}-4+L i_{2}(1)\right\}
$$

\section{A.3 Three-point integrals}

The three-point integrals will only be given for the special kinematics needed in the calculation, defined by the relations (63) to (65), since a general form depending on parameters like $\chi_{r}$ cannot be so neatly obtained as it was the case for the two-point integrals. Below we list the integrals in terms of form factors.

$$
\begin{aligned}
J_{3}^{F}(k, p) & =-Q_{\epsilon}^{k}\left(-k^{2}\right)^{-1} R_{0} \\
J_{3}^{F \mu}(k, p) & =Q_{\epsilon}^{k}\left(-k^{2}\right)^{-1}\left(R_{1} p^{\mu}+R_{2} k^{\mu}\right) \\
J_{3}^{F \mu \nu}(k, p) & =-Q_{\epsilon}^{k}\left(-k^{2}\right)^{-1}\left(R_{3} p^{\mu} p^{\nu}+R_{4} k^{\mu} k^{\nu}+R_{5}\{k p\}^{\mu \nu}+k^{2} R_{6} g^{\mu \nu}\right) \\
\{k p\}^{\mu \nu} & =k^{\mu} p^{\nu}+k^{\nu} p^{\mu} \\
J_{3}^{A}(k, p) & =\frac{Q_{\epsilon}^{k}}{p n}\left(-k^{2}\right)^{-1} S_{0} \\
J_{3 \mu}^{A}(k, p) & =-\frac{Q_{\epsilon}^{k}}{p n}\left(-k^{2}\right)^{-1}\left(S_{1} p_{\mu}+S_{2} k_{\mu}+S_{3} n_{\mu}+S_{4} n_{\mu}^{*}\right)
\end{aligned}
$$

Higher tensor integrals have been eliminated by Passarino-Veltman reduction.

\section{A.3.1 Form factors for the Feynman part}

\begin{tabular}{|c|c|c|c|c|c|c|}
\hline$R_{0}$ & $R_{1}$ & $R_{2}$ & $R_{3}$ & $R_{4}$ & $R_{5}$ & $R_{6}$ \\
\hline$\frac{1}{\epsilon^{2}}-\frac{\pi^{2}}{6}$ & $\frac{1}{\epsilon^{2}}+\frac{2}{\epsilon}+4-\frac{\pi^{2}}{6}$ & $-\left(\frac{1}{\epsilon}+2\right)$ & $R_{1}+\frac{1}{\epsilon}+3$ & $-\left(\frac{1}{2 \epsilon}+1\right)$ & $-\left(\frac{1}{2 \epsilon}+\frac{3}{2}\right)$ & $\frac{1}{4}\left(\frac{1}{\epsilon_{U V}}+3\right)$ \\
\hline
\end{tabular}

\section{A.3.2 Axial part form factors with ML prescription}

$$
\begin{aligned}
S_{0}^{M L} & =\frac{1}{\epsilon^{2}}+\frac{1}{\epsilon} \ln x-2 L i_{2}(1)-2 L i_{2}(1-x)-\frac{1}{2} \ln ^{2} x \\
S_{1}^{M L} & =\frac{1}{\epsilon^{2}}+\frac{1}{\epsilon}-\frac{1}{\epsilon} \frac{x \ln x}{1-x}-\frac{x \ln x}{1-x}-L i_{2}(1)+\frac{2 x}{1-x} L i_{2}(1-x)+\frac{1}{2} \frac{x}{1-x} \ln ^{2} x \\
S_{2}^{M L} & =\frac{1}{\epsilon} \frac{\ln x}{1-x}-\frac{2}{1-x} L i_{2}(1-x)-\frac{1}{2} \frac{\ln ^{2} x}{1-x} \\
S_{3}^{M L} & =-\frac{1}{2} \frac{k^{2}}{k n}\left(L i_{2}(1)-\frac{L i_{2}(1-x)}{1-x}\right)
\end{aligned}
$$




$$
S_{4}^{M L}=-\frac{1}{\epsilon}+\frac{x \ln x}{1-x}
$$

\section{A.3.3 Axial part form factors with PV prescription}

$$
\begin{aligned}
S_{0}^{P V} & =\frac{1}{\epsilon^{2}}-\frac{1}{\epsilon} I_{0}+\frac{1}{\epsilon} \ln x+I_{1}-I_{0} \ln x-2 L i_{2}(1)-2 L i_{2}(1-x)-\frac{1}{2} \ln ^{2} x \\
S_{1}^{P V} & =\frac{1}{\epsilon^{2}}-\frac{1}{\epsilon} \frac{x \ln x}{1-x}+\frac{x}{1-x} L i_{2}(1-x)-L i_{2}(1) \\
S_{2}^{P V} & =\frac{1}{\epsilon} \frac{\ln x}{1-x}-\frac{L i_{2}(1-x)}{1-x} \\
S_{3}^{P V} & =-\frac{1}{2} \frac{k^{2}}{k n}\left(\frac{I_{0}}{\epsilon}+\frac{1}{\epsilon} \frac{\ln x}{1-x}-I_{1}+\frac{I_{0} \ln x}{1-x}-L i_{2}(1)-\frac{x L i_{2}(1-x)}{1-x}+\frac{1}{2} \ln ^{2} x\right) \\
S_{4}^{P V} & =0
\end{aligned}
$$

\section{B Phase space parametrizations}

\section{B.1 Phase space integral for virtual contributions}

The phase space integral needed for the virtual diagrams, where only one gluon line is cut, is given by

$$
P S^{\text {virt }}=2 \pi z \int \frac{d^{m} k}{(2 \pi)^{m}} \delta(x-z) \delta\left((p-k)^{2}\right)
$$

where

$$
(p-k)^{2}=-\frac{k_{T}^{2}}{x}-\frac{(1-x)}{x} k^{2} \Longrightarrow \delta\left((p-k)^{2}\right)=x \delta\left(k_{T}^{2}+(1-x) k^{2}\right)
$$

Since the integrand has no angular dependence, the angular integral is trivial here, so

$$
\int d^{m} k=K_{m-2} \int d k^{2} \frac{d x}{2 x} d\left|k_{T}\right|\left|k_{T}\right|^{m-3}=K_{m-2} \int d k^{2} \frac{d x}{2 x} \frac{1}{2} d k_{T}^{2}\left(k_{T}^{2}\right)^{\frac{m-4}{2}}
$$

and

$$
K_{m-2}=\frac{2 \pi^{\frac{m-2}{2}}}{\Gamma\left(\frac{m-2}{2}\right)}
$$

is the surface of a $(m-2)$ dimensional hypersphere. Thus in $m=4-2 \epsilon$ dimensions, the phase space for the virtual diagrams is given by

$$
\begin{aligned}
P S^{v i r t} & =2 \pi z \int \frac{d^{m} k}{(2 \pi)^{m}} \delta(x-z) \delta\left((p-k)^{2}\right) \\
& =\frac{2 \pi}{(2 \pi)^{4-2 \epsilon}} \frac{1}{4} K_{2-2 \epsilon} \int d k^{2} d k_{T}^{2}\left(k_{T}^{2}\right)^{-\epsilon} x \delta\left(k_{T}^{2}+(1-x) k^{2}\right) \\
& =\frac{1}{16 \pi^{2}} \frac{(4 \pi)^{\epsilon}}{\Gamma(1-\epsilon)} \int_{0}^{Q^{2}} d\left(-k^{2}\right)\left(-k^{2}\right)^{-\epsilon} x(1-x)^{-\epsilon}
\end{aligned}
$$


The upper limit of the $d\left(-k^{2}\right)$ integral $\left(k^{2}<0\right)$ is denoted by a large momentum scale $Q^{2}$ whose actual value is irrelevant since only the pole part of the $k^{2}$-integration is needed.

\section{B.2 Phase space integral for axial ghost contributions}

The two-body-phase space where one of the cut lines, say $l_{2}$, is an axial ghost is goverened by the condition $l_{2}^{+}=0$

$$
\begin{aligned}
P S^{\text {ghost }}= & \frac{4 \pi^{2}}{(2 \pi)^{m}} \int d l_{1}^{+} d l_{2}^{+} d l_{1}^{-} d l_{2}^{-} d^{(m-2)} \vec{l}_{1} d^{(m-2)} \vec{l}_{2} d^{(m-2)} \vec{k}_{T} \delta\left(p^{+}-k^{+}-l_{1}^{+}-l_{2}^{+}\right) \\
& \cdot \delta^{(m-2)}\left(\vec{k}_{T}+\vec{l}_{1}+\vec{l}_{2}\right) \delta\left(k^{-}+l_{1}^{-}+l_{2}^{-}\right) \delta\left(l_{2}^{+}\right) \delta\left(l_{1}^{2}\right) \theta\left(l_{2}^{0}\right)
\end{aligned}
$$

Now use $\quad \delta\left(l_{1}^{2}\right)=\frac{1}{2 l_{1}^{+}} \delta\left(l_{1}^{-}-\frac{\vec{l}_{1}^{2}}{2 l_{1}^{+}}\right) \quad$ to eliminate $l_{1}^{-}$

substitute $l_{2}^{-}$by $\xi=2 p^{+} l_{2}^{-} \Rightarrow d l_{2}^{-}=\frac{d \xi}{2 p^{+}}$

Note that $\xi \geq 0 \quad$ because of the theta function $\theta\left(l_{2}^{0}\right)$ :

$$
\theta\left(l_{2}^{0}\right)=\theta\left(l_{2}^{+}+l_{2}^{-}\right)=\theta\left(l_{2}^{-}\right) \quad \text { for } l_{2}^{+}=0
$$

Hence

$$
\begin{aligned}
P S^{\text {ghost }=} & \frac{2 \pi^{2}}{(2 \pi)^{m}} \int \frac{d z_{1}}{z_{1}} d z_{2} \delta\left(z_{2}\right) \delta\left(1-x-z_{1}-z_{2}\right) \int d \vec{k}_{T} d \vec{l}_{1} d \vec{l}_{2} \delta\left(\vec{k}_{T}+\vec{l}_{1}+\vec{l}_{2}\right) \\
& \int_{0} d \xi \delta\left(\frac{k^{2}}{x}+\frac{\vec{k}_{T}^{2}}{x}+\xi+\frac{\vec{l}_{1}^{2}}{z_{1}}\right)
\end{aligned}
$$

For the diagram $\left(h^{D_{12}}\right)$, it is convenient to eliminate $\vec{l}_{2}$ by using the $\delta$-function for the transverse momenta. For diagrams $\left(b^{D_{12}}\right)$ and $\left(h^{D_{21}}\right)$ it is more convenient to eliminate $\vec{k}_{T}$, leading to a simpler form of the denominators. But the final form of the phase space of course is the same. So for definiteness, the substitutions suitable for topology $\left(h^{D_{12}}\right)$ will be given below.

The angle $\theta$ and the parameter $\beta$ are defined by

$$
\vec{k}_{T} \vec{l}_{1}=k_{T} l_{1} \cos \theta \quad ; \quad \beta^{2}=\frac{k_{T}^{2}}{l_{1}^{2}}
$$

Using

$$
\int d \vec{l}_{1} d \vec{k}_{T}=\frac{\pi^{2-2 \epsilon}}{\Gamma^{2}(1-\epsilon)} \int d l_{1}^{2}\left(l_{1}^{2}\right)^{-\epsilon} d k_{T}^{2}\left(k_{T}^{2}\right)^{-\epsilon} \cdot \frac{\Gamma(1-\epsilon)}{\sqrt{\pi} \Gamma\left(\frac{1}{2}-\epsilon\right)} \int_{0}^{\pi} d \theta(\sin \theta)^{-2 \epsilon}
$$

leads to

$$
P S^{\text {ghost }}=F_{\epsilon} \int \frac{d z_{1}}{z_{1}} \delta\left(1-x-z_{1}\right) \int d l_{1}^{2}\left(l_{1}^{2}\right)^{-\epsilon} d k_{T}^{2}\left(k_{T}^{2}\right)^{-\epsilon}
$$




$$
\begin{aligned}
& \cdot \int_{0} d \xi \delta\left(\frac{k^{2}}{x}+\frac{\vec{k}_{T}^{2}}{x}+\xi+\frac{\vec{l}_{1}^{2}}{z_{1}}\right) \cdot \frac{\Gamma(1-\epsilon)}{\sqrt{\pi} \Gamma\left(\frac{1}{2}-\epsilon\right)} \int_{0}^{\pi} d \theta(\sin \theta)^{-2 \epsilon} \\
F_{\epsilon}= & \frac{2 \pi^{4-2 \epsilon}}{(2 \pi)^{m}} \frac{1}{\Gamma^{2}(1-\epsilon)} .
\end{aligned}
$$

Now substitute

$$
k_{T}^{2}=\left|k^{2}\right| \cdot u \quad ; \quad l_{1}^{2}=\left|k^{2}\right| \frac{(1-x)}{x}(1-u) \cdot y
$$

then

$$
\beta^{2}=\frac{k_{T}^{2}}{l_{1}^{2}}=\frac{x u}{(1-x)(1-u) y} .
$$

This is leading to

$$
\begin{aligned}
P S^{\text {ghost }}= & F_{\epsilon}\left|k^{2}\right|^{2-2 \epsilon} x^{-1+\epsilon}(1-x)^{-\epsilon} \int_{0}^{1} d u u^{-\epsilon}(1-u)^{1-\epsilon} \int_{0}^{1} d y y^{-\epsilon} \\
& \cdot \int_{0} d \xi \delta\left(\frac{k^{2}}{x}(1-u)(1-y)+\xi\right) \cdot \frac{\Gamma(1-\epsilon)}{\sqrt{\pi} \Gamma\left(\frac{1}{2}-\epsilon\right)} \int_{0}^{\pi} d \theta(\sin \theta)^{-2 \epsilon} .
\end{aligned}
$$

Finally the integral over $\theta$ can be transformed to an integral from 0 to 1 by substituting $w=\frac{1}{2}(1+\cos \theta)$, leading to

$$
\begin{aligned}
P S^{\text {ghost }}= & F_{\epsilon}\left|k^{2}\right|^{2-2 \epsilon} x^{-1+\epsilon}(1-x)^{-\epsilon} \int_{0}^{1} d u u^{-\epsilon}(1-u)^{1-\epsilon} \int_{0}^{1} d y y^{-\epsilon} \\
& \frac{1}{B\left(\frac{1}{2}-\epsilon, \frac{1}{2}-\epsilon\right)} \int_{0}^{1} d w[w(1-w)]^{-\frac{1}{2}-\epsilon} .
\end{aligned}
$$

\section{References}

[1] R. K. Ellis, W. J. Stirling and B. R. Webber, QCD and Collider Physics, Cambridge, University Press (1996).

[2] S. G. Gorishny, A. L. Kataev and S. A. Larin, Phys. Lett. B259 (1991) 144;

M. A. Samuel and L. R. Surguladze Phys. Rev. Lett. 66 (1991) 560.

[3] S. A. Larin, et al., Nucl. Phys. B427 (1994) 41.

[4] R. Hamberg, W. L. van Neerven and T. Matsuura, Nucl. Phys. B359 (1991) 343.

[5] E. G. Floratos, D. A. Ross and C. T. Sachrajda, Nucl. Phys. B129 (1977) 66; E: Nucl. Phys. B139 (1978) 545 545; Nucl. Phys. B152 (1979) 493;

A. Gonzalez-Arroyo, C. Lopez and F. J. Yndurain, Nucl. Phys. B153 (1979) 161;

E. G. Floratos, R. Lacaze and C. Kounnas, Nucl. Phys. B192 (1981) 417. 
[6] G. Curci, W. Furmanski and R .Petronzio, Nucl. Phys. B175 (1980) 27.

[7] W. Furmanski and R. Petronzio, Phys. Lett. 97B (1980) 437.

[8] R. Mertig and W. L. van Neerven, Z. Phys. C70 (1996) 637.

[9] W. Vogelsang, Nucl. Phys. B475 (1996) 47 and W. Vogelsang, Phys. Rev. D54 (1996) 2023.

[10] J. C. Collins and D. E. Soper, Nucl. Phys. B194 (1982) 445.

[11] J. C. Collins and R. J. Scalise, Phys. Rev. D50 (1994) 4117.

[12] R. K. Ellis and W. Vogelsang, hep-ph/9602356.

[13] R. K. Ellis, H. Georgi, M. Machacek, H. D. Politzer and G. G. Ross, Nucl. Phys. B152 (1979) 285.

[14] S. Mandelstam, Nucl. Phys. B213 (1983) 149.

[15] G. Leibbrandt, Phys. Rev. D29 (1984) 1699.

[16] G. Leibbrandt, Rev. Mod. Phys. 59 (1987) 1067.

[17] A. Bassetto, G. Nardelli, R. Soldati, Yang-Mills theories in algebraic non-covariant gauges, World Scientific, Singapore 1991.

[18] G. Leibbrandt, Noncovariant gauges: Quantization of Yang-Mills and Chern-Simons theory in axial type gauges, World Scientific, Singapore 1994.

[19] A. Bassetto, Phys. Rev. D47 (1993) 727;

A. Bassetto, Nucl. Phys. Proc. Suppl. 51C (1996) 281.

[20] A. Bassetto, M. Dalbosco, I. Lazzizzera and R.Soldati, Phys. Rev. D31 (1985) 2012.

[21] G. McCartor, D.G. Robertson, Z. Phys. C62 (1994) 349.

[22] M. Oegren, Classical and Quantum Gravity, No. 3 (1986) 581.

[23] D. M. Capper, J. J. Dulwich and M. J. Litvak, Nucl. Phys. B241 (1984) 463. 